Article

\title{
Bonferroni Mean Operators of Linguistic Neutrosophic Numbers and Their Multiple Attribute Group Decision-Making Methods
}

\author{
Changxing Fan ${ }^{1, *}$, Jun $\mathrm{Ye}^{2} \mathbb{B}^{\mathbb{D}}$, Keli Hu ${ }^{1}$ and En Fan ${ }^{1}$ \\ 1 Department of Computer Science, Shaoxing University, 508 Huancheng West Road, \\ Shaoxing 312000, Zhejiang, China; ancimoon@gmail.com (K.H.); efan@usx.edu.cn (E.F.) \\ 2 Department of Electrical and Information Engineering, Shaoxing University, 508 Huancheng West Road, \\ Shaoxing 312000, Zhejiang, China; yehjun@aliyun.com \\ * Correspondence: fcxjszj@usx.edu.cn; Tel.: +86-575-8820-2669
}

Received: 11 August 2017; Accepted: 31 August 2017; Published: 1 September 2017

\begin{abstract}
Linguistic neutrosophic numbers (LNN) is presented by Fang and Ye in 2017, which can describe the truth, falsity, and indeterminacy linguistic information independently. In this paper, the LNN and the Bonferroni mean operator are merged together to propose a LNN normalized weighted Bonferroni mean (LNNNWBM) operator and a LNN normalized weighted geometric Bonferroni mean (LNNNWGBM) operator and the properties of these two operators are proved. Further, multi-attribute group decision methods are introduced based on the proposed LNNNWBM and LNNNWGBM operators, and then an example is provided to demonstrate the application and validity of the proposed methods. In addition, in order to consider the effect of the parameters $p$ and $q$ on the decision results, different pairs of parameter values are employed to verify the decision results.

Keywords: linguistic neutrosophic numbers (LNN); LNN normalized weighted Bonferroni mean (LNNNWBM) operator; LNN normalized weighted geometric Bonferroni mean (LNNNWGBM) operator; group decision-making
\end{abstract}

\section{Introduction}

In dealing with the complex, unknown, and uncertain decision-making problems, a group of decision-makers are usually employed to analyze a set of alternatives and to get the optimal result in a certain way. Such a decision-making process is called multiple attribute group decision-making (MAGDM) problem. When making decisions, decision-makers tend to use words such as "excellent", "good", and "poor" to express their evaluations for objects. Zadeh proposed a linguistic variable set $S=\left\{S_{0}, S_{1}, S_{2}, S_{3}, \ldots, S_{g}\right\}$ (g is an even number) to deal with the approximate reasoning problems $[1,2]$. The linguistic variable is an effective tool, it improves the reliability and flexibility of classical decision models $[3,4]$. In recent years, the linguistic variables have been frequently linked to other theories. Liu proposed the intuitionistic linguistic set (ILS) composed of linguistic variables and IFS, where the first component provides its qualitative evaluation value/linguistic value and the second component gives the credibility of its intuitionistic fuzzy value for the given linguistic value [5]. Then, Chen et al. proposed the linguistic intuitionistic fuzzy number (LIFN), which is composed of the intuitionistic fuzzy number (the basic element in IFS) and the linguistic variable [6]. On the other hand, some methods for multiple attribute group decision-making (MAGDM) were proposed based on two-dimension uncertain linguistic variable $[7,8]$. Some improved linguistic intuitionistic fuzzy aggregation operators and several corresponding applications were given in decision-making [9]. Although the IFS theory considers not only $T(x)$, but also $F(x)$, IFS is still not perfect enough because it ignores the indeterminate and inconsistent information. Thus, the intuitionistic fuzzy number can only 
be used for expressing incomplete information, but not for expressing indeterminate and inconsistent information. To make up for the insufficiency of the IFS theory, Smarandache put forward the neutrosophic set (NS) composed of three parts: truth $T(x)$, falsity $F(x)$, and indeterminacy $I(x)[10,11]$. Wang et al. and Smarandache also proposed the concept of a single-valued neutrosophic set (SVNS) satisfying $T(x), I(x), F(x) \subseteq[0,1], 0 \leq T(x)+F(x)+I(x) \leq 3$ [10-12]. Ye proposed an extended TOPSIS (technique for order preference by similarity to an ideal Solution) method for MAGDM based on single valued neutrosophic linguistic numbers (SVNLNs), which are basic elements in a single-valued neutrosophic linguistic set (SVNLS) [13]. Liu and Shi presented some neutrosophic uncertain linguistic number Heronian mean operators and their application to MAGDM [14]. Since the Bonferroni mean (BM) is a useful operator in decision-making [15], it was extended to hesitant fuzzy sets, IFSs, and interval-valued IFSs to propose their some Bonferroni mean operators for decision making [16-20]. Then, Fang and Ye proposed the linguistic neutrosophic numbers (LNN) and their basic operational laws [21]. LNN consists of the truth, indeterminacy, and falsity linguistic degrees, which can be expressed as the form $a=\left\langle l_{T}, l_{I}, l_{F}\right\rangle$, but the LIFN and SVNLN cannot express such linguistic evaluation value. In [21], Fang and Ye also presented a LNN-weighted arithmetic averaging (LNNWAA) operator and a LNN-weighted geometric averaging (LNNWGA) operator for MAGDM. However, the Bonferroni mean operator is not extended to LNNs so far. Hence, this paper proposes a LNN normalized weighted Bonferroni mean (LNNNWBM) operator, a LNN normalized weighted geometric Bonferroni mean (LNNNWGBM) operator and their MAGDM methods. Compared with the aggregation operators in $[14,21]$, the LNNNWBM and LNNNWGBM operators can calculate the final weights by the relation between attribute values, which can make the information aggregation more objective and reliable.

The rest organizations of this paper are as follows. Section 2 describes some basic concepts of LNN, the basic operational laws of LNNs, and the basic concepts of BM and the normalized weighted BM. Section 3 proposes the LNNNWBM and LNNNWGBM operators and investigates their properties. Section 4 establishes MAGDM methods by using the LNNNWBM operator and LNNNWGBM operator. Section 5 provides an illustrative example with different values of the parameters $p$ and $q$ to demonstrate the application of the proposed methods. Section 6 gives conclusions.

\section{Some Concepts of LNNs and BM}

\subsection{Linguistic Neutrosophic Numbers and Their Operational Laws}

Definition 1 [21]. Set $L=\left\{l_{0}, l_{1}, l_{2}, \ldots, l_{g}\right\}$ as a language term set, in which $g$ is an even number and $g+1$ is the particle size of $L$. If $a=\left\langle l_{T}, l_{I}, l_{F}\right\rangle$ is defined for $l_{T}, l_{I}, l_{F} \in L$ and $T, I, F \in[0, g]$, where $l_{T}$ expresses the truth degree, $l_{I}$ expresses indeterminacy degree, and $l_{F}$ expresses falsity degree by linguistic terms, then $a$ is called an LNN.

Definition 2 [21]. Set $a=\left\langle l_{T}, l_{1}, l_{F}\right\rangle, a_{1}=\left\langle l_{T_{1}}, l_{I_{1}}, l_{F_{1}}\right\rangle$, and $a_{2}=\left\langle l_{T_{2}}, l_{I_{2}}, l_{F_{2}}\right\rangle$ as three LNNs in $L$, the number $\lambda \geq 0$, they have the follow operational laws:

$$
\begin{gathered}
a_{1} \oplus a_{2}=\left\langle l_{T_{1}}, l_{I_{1}}, l_{F_{1}}\right\rangle \oplus\left\langle l_{T_{2}}, l_{I_{2}}, l_{F_{2}}\right\rangle=\left\langle l_{T_{1}+T_{2}-\frac{T_{1} T_{2}}{g}}, \frac{l_{I_{1} I_{2}}}{g}, \frac{l_{F_{1} F_{2}}}{g}\right\rangle ; \\
a_{1} \otimes a_{2}=\left\langle l_{T_{1}}, l_{I_{1}}, l_{F_{1}}\right\rangle \otimes\left\langle l_{T_{2}}, l_{I_{2}}, l_{F_{2}}\right\rangle=\left\langle l_{\frac{T_{1} T_{2}}{g}}, l_{I_{1}+I_{2}-\frac{I_{1} I_{2}}{g}}, l_{F_{1}+F_{2}-\frac{F_{1} F_{2}}{g}}\right\rangle ; \\
\lambda a=\lambda\left\langle l_{T}, l_{1}, l_{F}\right\rangle=\left\langle l_{g-g\left(1-\frac{T}{g}\right)^{\lambda}}, l_{g\left(\frac{I}{g}\right)^{\lambda}}, l_{g\left(\frac{F}{g}\right)^{\lambda}}\right\rangle ; \\
a^{\lambda}=\left\langle l_{T}, l_{1}, l_{F}\right\rangle^{\lambda}=\left\langle l_{g\left(\frac{T}{g}\right)^{\lambda}}, l_{g-g\left(1-\frac{I}{g}\right)^{\lambda}}, l_{g-g\left(1-\frac{F}{g}\right)^{\lambda}}\right\rangle .
\end{gathered}
$$


Definition 3 [21]. Set $a=\left\langle l_{T}, l_{I}, l_{F}\right\rangle$ as an LNN in $L$, then the expectation $E(a)$ and the accuracy $H(a)$ can be defined as follows:

$$
\begin{gathered}
E(a)=(2 g+T-I-F) / 3 g \\
H(a)=(T-F) / g
\end{gathered}
$$

Definition 4 [21]. Set $a_{1}=\left\langle l_{T_{1}}, l_{I_{1}}, l_{F_{1}}\right\rangle$ and $a_{2}=\left\langle l_{T_{2}}, l_{I_{2}}, l_{F_{2}}\right\rangle$ as two LNNs, then:

If $E\left(a_{1}\right)>E\left(a_{2}\right)$, then $a_{1} \succ a_{2}$;

If $E\left(a_{1}\right)=E\left(a_{2}\right)$ then

If $H\left(a_{1}\right)>H\left(a_{2}\right)$, then $a_{1} \succ a_{2}$;

If $H\left(a_{1}\right)=H\left(a_{2}\right)$, then $a_{1} \sim a_{2}$;

If $H\left(a_{1}\right)<H\left(a_{2}\right)$, then $a_{1} \prec a_{2}$.

2.2. Bonferroni Mean Operators

Definition 5 [15]. Let $\left(a_{1}, a_{2}, \ldots, a_{n}\right)$ be a set of non-negative numbers, the function BM: $R^{n} \rightarrow R$. If $p, q \geq 0$ and BM satisfies:

$$
B M^{p, q}\left(a_{1}, a_{2}, \ldots, a_{n}\right)=\left(\frac{1}{n(n-1)} \sum_{\substack{i, j=1 \\ j \neq i}}^{n} a_{i}^{p} a_{j}^{q}\right)^{\frac{1}{p+q}}
$$

then $B M^{p, q}$ is called a $B M$ operator.

Definition 6 [16]. Let $\left(a_{1}, a_{2}, \ldots, a_{n}\right)$ be a set of non-negative numbers, the function NWBM: $R^{n} \rightarrow R$, $w_{i}(i=1,2, \ldots, n)$ be the relative weight of $a_{i}(i=1,2, \ldots, n), w_{i} \in[0,1]$, and $\sum_{i=1}^{n} w_{i}=1$. If $p, q \geq 0$ and NWBM satisfies:

$$
\operatorname{NWBM}^{p, q}\left(a_{1}, a_{2}, \ldots, a_{n}\right)=\left(\sum_{\substack{i, j=1 \\ j \neq i}}^{n} \frac{w_{i} w_{j}}{1-w_{i}} a_{i}{ }^{p} a_{j}\right)^{\frac{1}{p+q}}
$$

then $N W B M^{p, q}$ is called a normalized weighted BM operator.

Definition 7 [17]. Let $\left(a_{1}, a_{2}, \ldots, a_{n}\right)$ be a set of non-negative numbers, the function GBM: $R^{n} \rightarrow R$. If $p, q \geq 0$ and GBM satisfies:

$$
\operatorname{GBM}^{p, q}\left(a_{1}, a_{2}, \ldots, a_{n}\right)=\left(\frac{1}{n} \sum_{i}^{n} a_{i}^{p}\left(\prod_{j=1, j \neq i}^{n} a_{j}^{q}\right)^{\frac{1}{n-1}}\right)^{\frac{1}{p+q}}
$$

then $G B M^{p, q}$ is called a geometric BM operator.

Definition 8 [18-20]. Let $\left(a_{1}, a_{2}, \ldots a_{n}\right)$ be a set of non-negative numbers, the function NWGBM: $R^{n} \rightarrow R, w_{i}$ $(i=1,2, \ldots, n)$ be the relative weight of $a_{i}(i=1,2, \ldots, n), w_{i} \in[0,1]$, and $\sum_{i=1}^{n} w_{i}=1$. If $p, q \geq 0$ and NWGBM satisfies:

$$
\operatorname{NWGBM}^{p, q}\left(a_{1}, a_{2}, \ldots, a_{n}\right)=\frac{1}{p+q} \otimes_{i, j=1, j \neq i}^{n}\left(p a_{i} \oplus q a_{j}\right)^{\frac{w_{i} w_{j}}{1-w_{i}}}
$$

then NWGBM $M^{p, q}$ is called a normalized weighted geometric BM (NWGBM) operator. 


\section{Two BM Aggregation Operators of LNNs}

\subsection{Normalized Weighted BM Operators of LNNs}

Definition 9. Set $a_{i}=\left\langle l_{T_{i}}, l_{I_{i}}, l_{F_{i}}\right\rangle(i=1,2, \ldots, n)$ as a collection of LNNs in $L$, then the LNNNWBM operator can be defined as follows:

$$
\operatorname{LNNNWBM}^{p, q}\left(a_{1}, a_{2}, \ldots, a_{n}\right)=\left(\sum_{\substack{i, j=1 \\ j \neq i}}^{n} \frac{w_{i} w_{j}}{1-w_{i}} a_{i}^{p} a_{j}^{q}\right)^{\frac{1}{p+q}}
$$

where $w_{i}$ is the relative weight of $a_{i}, w_{i} \in[0,1]$, and $\sum_{i=1}^{n} w_{i}=1, w_{j}$ is the relative weight of $a_{j}, w_{j} \in[0,1]$, and $\sum_{j=1}^{n} w_{j}=1$.

According to Definitions 2 and 9, we can get the following theorem:

Theorem 1. Set $a_{i}=\left\langle l_{T_{i}}, l_{I_{i}}, l_{F_{i}}\right\rangle(i=1,2, \ldots, n)$ as a collection of LNNs in $L$, then by the Equation (11), the aggregation result obtained is still an $L N N$, and we can get the following aggregation formula:

$$
\begin{aligned}
& \operatorname{LNNNWBM} M^{p, q}\left(a_{1}, a_{2}, \ldots, a_{n}\right)=\left(\sum_{\substack{i j=1 \\
j \neq i}}^{n w_{i} w_{j}} a_{i} a_{i}^{p} a_{j}\right)^{\frac{1}{p+q}}
\end{aligned}
$$

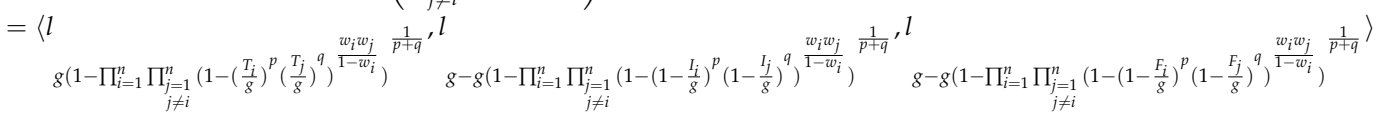

where $w_{i}$ is the relative weight of $a_{i}, w_{i} \in[0,1]$, and $\sum_{i=1}^{n} w_{i}=1, w_{j}$ is the relative weight of $a_{j}, w_{j} \in[0,1]$, and $\sum_{j=1}^{n} w_{j}=1$.

\section{Proof 1.}

(1) $\quad a_{i}^{p}=\left\langle l_{g\left(\frac{T_{i}}{g}\right)^{p}} l_{g-g\left(1-\frac{I_{i}}{g}\right)^{p,}} l_{g-g\left(1-\frac{F_{i}}{g}\right)^{p}}\right\rangle$;

(2) $a_{j}{ }^{q}=\left\langle l_{g\left(\frac{T_{j}}{g}\right)^{q,} l} l_{g-g\left(1-\frac{I_{j}}{g}\right)^{q,} l} g-g\left(1-\frac{F_{j}}{g}\right)^{q}\right.$;

$$
a_{i}^{p} \otimes a_{j}^{q}
$$

$$
\begin{aligned}
& =\left\langle l_{g\left(\frac{T_{i}}{8}\right)^{p} g\left(\frac{T_{j}}{8}\right)}\right),
\end{aligned}
$$

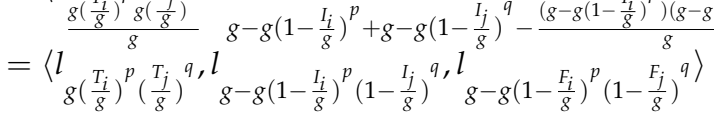

(4)

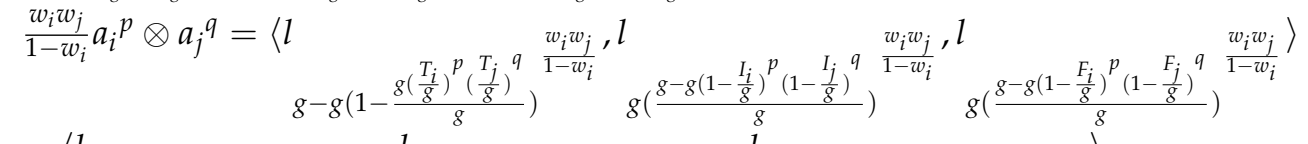

$$
\begin{aligned}
& =\left\langle l \mid g-g\left(1-\left(\frac{T_{i}}{g}\right)^{p}\left(\frac{T_{j}}{g}\right)^{q}\right)^{q \frac{w_{i} w_{j}}{1-w_{i}}, l} g\left(1-\left(1-\frac{I_{i}}{g}\right)^{p}\left(1-\frac{I_{j}}{g}\right)^{q}\right)^{q \frac{w_{i} w_{j}}{1-w_{i}}, l} g\left(1-\left(1-\frac{F_{i}}{g}\right)^{p}\left(1-\frac{F_{j}}{g}\right)^{q}\right)^{q \frac{w_{i} w_{j}}{1-w_{i}}}\right\rangle^{g} \\
& \oplus_{i=1}^{n} \oplus_{j=1, j \neq i}^{n} \frac{w_{i} w_{j}}{1-w_{i}} a_{i}{ }^{p} \otimes a_{j}{ }^{q}
\end{aligned}
$$

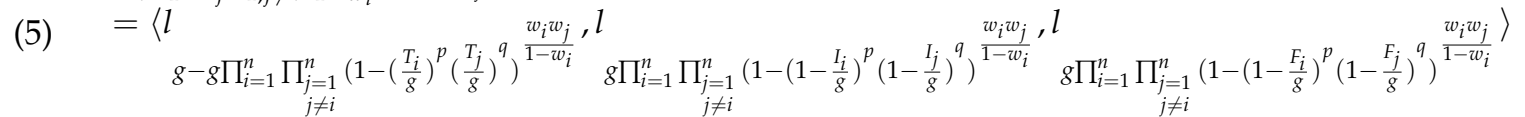

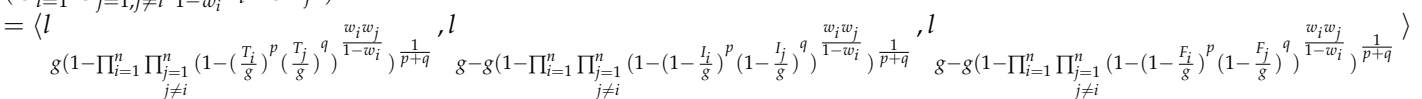

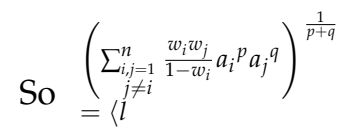

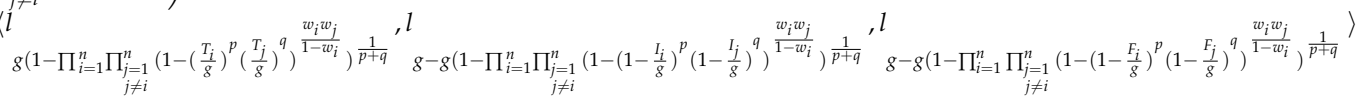

The proof of Theorem 1 is completed. 
Theorem 2. (Idempotency). Set $a_{i}=\left\langle l_{T_{i}}, l_{I_{i}}, l_{F_{i}}\right\rangle(i=1,2, \ldots, n)$ as a collection of LNNs in $L$, if $a_{i}=a$, then

$$
\operatorname{LNNNWBM}^{p, q}\left(a_{1}, a_{2}, \ldots, a_{n}\right)=\operatorname{LNNNWBM} M^{p, q}(a, a, \ldots, a)=a .
$$

Proof 2. Since $a_{i}=a$, i.e., $T_{i}=T ; I_{i}=I ; F_{i}=F$ for $i=1,2, \ldots, n$, there are the following result:

$$
\begin{aligned}
& \operatorname{LNNNWBM} M^{p, q}\left(a_{1}, a_{2}, \ldots, a_{n}\right)=\operatorname{LNNNWBM} M^{p, q}(a, a, \ldots, a)=\left(\sum_{\substack{i, j=1 \\
j \neq i}}^{n} \frac{w_{i} w_{j}}{1-w_{i}} a^{p} a^{q}\right)^{\frac{1}{p+q}}
\end{aligned}
$$

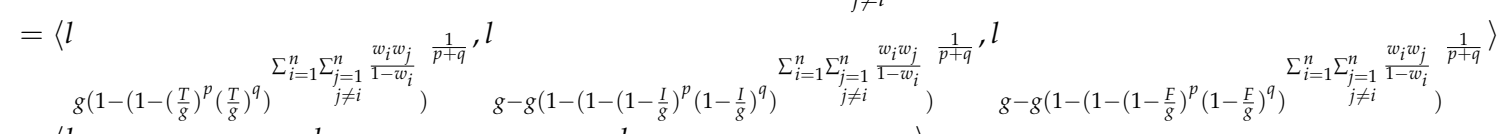

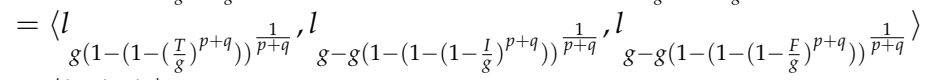

$$
\begin{aligned}
& =\left\langle l_{T}, l_{I}, l_{F}\right\rangle=a \text {. }
\end{aligned}
$$

The proof of Theorem 2 is completed.

Theorem 3. (Monotonicity). Set $a_{i}=\left\langle l_{T_{i^{\prime}}}, l_{I_{i}}, l_{F_{i}}\right\rangle$ and $b_{i}=\left\langle l_{T_{i}}, l_{I_{i}}, l_{F_{i}}\right\rangle(i=1,2, \ldots, n)$ as two collections of LNNs in $L$, if $T_{i} \leq T_{i}{ }^{\prime}, I_{i} \geq I_{i}{ }^{\prime}$, and $F_{i} \geq F_{i}{ }^{\prime}$ then $\operatorname{LNNNWBM}^{p, q}\left(a_{1}, a_{2}, \ldots, a_{n}\right) \leq$ $\operatorname{LNNNWBM} M^{p, q}\left(b_{1}, b_{2}, \ldots, b_{n}\right)$.

Proof 3. Since $T_{i} \leq T_{i}{ }^{\prime}, I_{i} \geq I_{i}{ }^{\prime}$ and $F_{i} \geq F_{i}{ }^{\prime}$, we can easy obtain:

$$
\begin{aligned}
& 1-\left(\frac{T_{i}}{g}\right)^{p}\left(\frac{T_{j}}{g}\right)^{q} \geq 1-\left(\frac{T_{i} \prime}{g}\right)^{p}\left(\frac{T_{j} \prime}{g}\right)^{q} \\
& 1-\prod_{i=1}^{n} \prod_{\substack{j=1 \\
j \neq i}}^{n}\left(1-\left(\frac{T_{i}}{g}\right)^{p}\left(\frac{T_{j}}{g}\right)^{q}\right)^{\frac{v_{i} w_{j}}{1-w_{i}}} \leq 1-\prod_{i=1}^{n} \prod_{\substack{j=1 \\
j \neq i}}^{n}\left(1-\left(\frac{T_{i} \prime}{g}\right)^{p}\left(\frac{T_{j}{ }^{\prime}}{g}\right)^{q}\right)^{\frac{w_{i} w_{j}}{1-w_{i}}}, \\
& g\left(1-\prod_{i=1}^{n} \prod_{\substack{j=1 \\
j \neq i}}^{n}\left(1-\left(\frac{T_{i}}{g}\right)^{p}\left(\frac{T_{j}}{g}\right)^{q}\right)^{\frac{w_{i} w_{j}}{1-w_{i}}}\right)^{\frac{1}{p+q}} \leq g\left(1-\prod_{i=1}^{n} \prod_{\substack{j=1 \\
j \neq i}}^{n}\left(1-\left(\frac{T_{i}{ }^{\prime}}{g}\right)^{p}\left(\frac{T_{j}{ }^{\prime}}{g}\right)^{q}\right)^{\frac{w_{i} w_{j}}{1-w_{i}}}\right)^{\frac{1}{p+q}} .
\end{aligned}
$$

Similarly

$$
\begin{aligned}
& \left(1-\frac{I_{i}}{g}\right)^{p}\left(1-\frac{I_{j}}{g}\right)^{q} \leq\left(1-\frac{I_{i}{ }^{\prime}}{g}\right)^{p}\left(1-\frac{I_{j} w_{j}}{g}\right)^{q}, \\
& 1-\prod_{i=1}^{n} \prod_{\substack{j=1 \\
j \neq i}}^{n}\left(1-\left(1-\frac{I_{i}}{g}\right)^{p}\left(1-\frac{I_{j}}{g}\right)^{q}\right)^{\frac{w_{i} w_{j}}{1-w_{i}}} \leq 1-\prod_{i=1}^{n} \prod_{\substack{j=1 \\
j \neq i}}^{n}\left(1-\left(1-\frac{I_{i}{ }^{\prime}}{g}\right)^{p}\left(1-\frac{I_{j}^{\prime}}{g}\right)^{q}\right)^{\frac{w_{i} w_{j}}{1-w_{i}}}, \\
& g-g\left(1-\prod_{i=1}^{n} \prod_{\substack{j=1 \\
j \neq i}}^{n}\left(1-\left(1-\frac{I_{i}}{g}\right)^{p}\left(1-\frac{I_{j}}{g}\right)^{q}\right)^{\frac{w_{i} w_{j}}{1-w_{i}}}\right)^{\frac{1}{p+q}} \\
& \geq g-g\left(1-\prod_{i=1}^{n} \prod_{\substack{j=1 \\
j \neq i}}^{n}\left(1-\left(1-\frac{I_{i} \prime}{g}\right)^{p}\left(1-\frac{I_{j} \prime}{g}\right)^{q}\right)^{\frac{w_{i} w_{j}}{1-w_{i}}}\right)^{\frac{1}{p+q}}
\end{aligned}
$$

and

$$
\begin{aligned}
& g-g\left(1-\prod_{i=1}^{n} \prod_{\substack{j=1 \\
j \neq i}}^{n}\left(1-\left(1-\frac{F_{i}}{g}\right)^{p}\left(1-\frac{F_{j}}{g}\right)^{q}\right)^{\frac{w_{i} w_{j}}{1-w_{i}}}\right)^{\frac{1}{p+q}} \\
\geq & g-g\left(1-\prod_{i=1}^{n} \prod_{\substack{j=1 \\
j \neq i}}^{n}\left(1-\left(1-\frac{F_{i} \prime}{g}\right)^{p}\left(1-\frac{F_{j} \prime}{g}\right)^{q}\right)^{\frac{w_{i} w_{j}}{1-w_{i}}}\right)^{\frac{1}{p+q}} .
\end{aligned}
$$

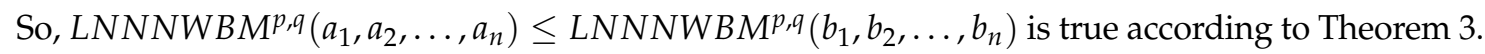
Therefore, the proof of Theorem 3 is completed. 
Theorem 4. (Boundedness). Set $a_{i}=\left\langle l_{T_{i}}, l_{I_{i}}, l_{F_{i}}\right\rangle(i=1,2, \ldots, n)$ as a collections of $L N N s$ in $L$, let $a^{-}=$ $\left\langle\min \left(l_{T_{i}}\right), \max \left(l_{I_{i}}\right), \max \left(l_{F_{i}}\right)\right\rangle$ and $a^{+}=\left\langle\max \left(l_{T_{i}}\right), \min \left(l_{I_{i}}\right), \min \left(l_{F_{i}}\right)\right\rangle$, then:

$$
a^{-} \leq \operatorname{LNNNWBM}^{p, q}\left(a_{1}, a_{2}, \ldots, a_{n}\right) \leq a^{+}
$$

Proof 4. According Theorem 2, we can obtain:

$a^{-}=\operatorname{LNNNWB} B^{p, q}\left(a^{-}, a^{-} \ldots a^{-}\right)$and $a^{+}=\operatorname{LNNNWBM} M^{p, q}\left(a^{+}, a^{+} \ldots a^{+}\right)$

According Theorem3, we can obtain:

$\operatorname{LNNNWBM}^{p, q}\left(a^{-}, a^{-} \ldots a^{-}\right) \leq \operatorname{LNNNWBM}^{p, q}\left(a_{1}, a_{2}, \ldots, a_{n}\right) \leq \operatorname{LNNNWBM}^{p, q}\left(a^{+}, a^{+} \ldots a^{+}\right)$.

Then $a^{-} \leq \operatorname{LNNNWBM} M^{p, q}\left(a_{1}, a_{2}, \ldots, a_{n}\right) \leq a^{+}$.

The proof of Theorem 4 is completed.

3.2. Normalized Weighted Geometric BM Operators of LNNs

Definition 10. Set $a_{i}=\left\langle l_{T_{i}}, l_{I_{i}}, l_{F_{i}}\right\rangle(i=1,2, \ldots, n)$ as a collection of LNNs in $L$, then the LNNNWGBM operator can be defined as follows:

$$
\operatorname{LNNNWGBM} M^{p, q}\left(a_{1}, a_{2}, \ldots, a_{n}\right)=\frac{1}{p+q} \otimes_{i=1}^{n} \otimes_{j=1, j \neq i}^{n}\left(p a_{i} \oplus q a_{j}\right)^{\frac{w_{i} w_{j}}{1-w_{i}}}
$$

where $w_{i}$ is the relative weight of $a_{i}, w_{i} \in[0,1]$, and $\sum_{i=1}^{n} w_{i}=1, w_{j}$ is the relative weighted of $a_{j}, w_{j} \in[0,1]$, and $\sum_{j=1}^{n} w_{j}=1$.

According to Definitions 2 and 10, we can get the following theorem:

Theorem 5. Set $a_{i}=\left\langle l_{T_{i}}, l_{I_{i}}, l_{F_{i}}\right\rangle(i=1,2, \ldots, n)$ as a collection of LNNs in $L$, then by the Equation (13) the aggregation result obtained is still an $L N N$, and we can get the following aggregation formula:

$$
\begin{aligned}
& \operatorname{LNNNWGBM} M^{p, g}\left(a_{1}, a_{2}, \ldots, a_{n}\right)=\frac{1}{p+q} \oplus_{i=1}^{n} \oplus_{j=1, j \neq i}^{n}\left(p a_{i} \oplus q a_{j}\right)^{\frac{w_{i} w_{j}}{1-w_{i}}}
\end{aligned}
$$

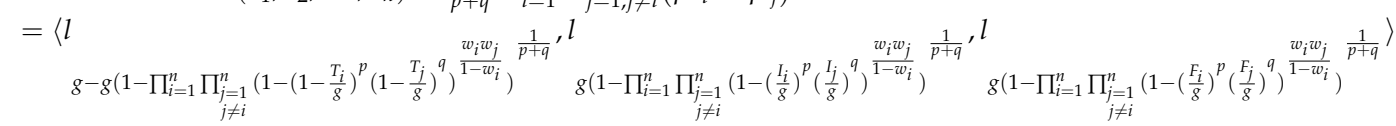

where $w_{i}$ is the relative weight of $a_{i}, w_{i} \in[0,1]$, and $\sum_{i=1}^{n} w_{i}=1, w_{j}$ is the relative weighted of $a_{j}, w_{j} \in[0,1]$, and $\sum_{j=1}^{n} w_{j}=1$.

The proof of Theorem 5 is similar to that of Theorem 1, so we do not repeat it again.

Theorem 6. (Idempotency). Set $a_{i}=\left\langle l_{T_{i}}, l_{i_{i}}, l_{F_{i}}\right\rangle(i=1,2, \ldots, n)$ as a collection of $L N N s$ in $L$, if $a_{i}=a$, then

$$
\operatorname{LNNNWGBM} M^{p, g}\left(a_{1}, a_{2}, \ldots, a_{n}\right)=\operatorname{LNNNWGBM} M^{p, g}(a, a \ldots a)=a
$$

The proof of Theorem 6 is similar to that of Theorem 2, so we don't repeat it again.

Theorem 7. (Monotonicity). Set $a_{i}=\left\langle l_{T_{i^{\prime}}}, l_{I_{i^{\prime}}}, l_{F_{i}}\right\rangle$ and $b_{i}=\left\langle l_{T_{i^{\prime}},}, l_{I_{i}{ }^{\prime}}, l_{F_{i}{ }^{\prime}}\right\rangle(i=1,2, \ldots, n)$ as two collections of LNNs in $L$, if $T_{i} \leq T_{i}{ }^{\prime}, I_{i} \geq I_{i}{ }^{\prime}$ and $F_{i} \geq F_{i}{ }^{\prime}$ then:

$$
\operatorname{LNNNWGBM}^{p, q}\left(a_{1}, a_{2}, \ldots, a_{n}\right) \leq \operatorname{LNNNWGBM}^{p, q}\left(b_{1}, b_{2}, \ldots, b_{n}\right)
$$

The proof of Theorem 7 is similar to that of Theorem 3, so we do not repeat it again. 
Theorem 8. (Boundedness). Set $a_{i}=\left\langle l_{T_{i}}, l_{I_{i}}, l_{F_{i}}\right\rangle(i=1,2, \ldots, n)$ as a collections of $L N N s$ in $L$, let $a^{-}=$ $\left\langle\min \left(l_{T_{i}}\right), \max \left(l_{I_{i}}\right), \max \left(l_{F_{i}}\right)\right\rangle$ and $a^{+}=\left\langle\max \left(l_{T_{i}}\right), \min \left(l_{I_{i}}\right), \min \left(l_{F_{i}}\right)\right\rangle$, then:

$$
a^{-} \leq \operatorname{LNNNWGBM} M^{p, q}\left(a_{1}, a_{2}, \ldots, a_{n}\right) \leq a^{+}
$$

The proof of Theorem 8 is similar to that of Theorem 4, so we do not repeat it again.

\section{MAGDM Methods Based on the LNNNWBM or LNNNWGBM Operator}

In this section, we will use the LNNNWBM or LNNNWGBM operator to deal with the MAGDM problems with LNN information.

In a MAGDM problem, there is a set of several alternatives $A=\left\{A_{1}, A_{2}, \ldots, A_{m}\right\}$ with a set of some attributes $C=\left\{C_{1}, C_{2}, \ldots, C_{n}\right\}$. Then, $\lambda=\left(\lambda_{1}, \lambda_{2}, \ldots, \lambda_{n}\right)^{T}$ with $\lambda_{i} \geq 0$ and $\sum_{i=1}^{n} \lambda_{i}=1$ are the weights of $C_{i}(i=1,2, \ldots, n)$. Now, there is a set of $t$ experts $E=\left\{E_{1}, E_{2}, \ldots, E_{t}\right\}$ to evaluate the MAGDM problem. Assume that $w=\left(w_{1}, w_{2}, \ldots, w_{t}\right)^{T}$ with $w_{j} \geq 0$ and $\sum_{j=1}^{t} w_{j}=1$ is the vector of the weights for $E_{y}(y=1,2, \ldots, t)$ and $L=\left\{l_{1}, l_{2}, \ldots, l_{g}\right\}$ is the given linguistic term set. The assessed value of the expert $E_{y}$ for $A_{i}$ with attribute $C_{j}$ is $a_{i j}^{(y)}=\left\langle l_{T_{i j}}^{y}, l_{I_{i j}}^{y}, l_{F_{i j}}^{y}\right\rangle \in$ $A(y=1,2, \ldots, t ; i=1,2, \ldots, m ; j=1,2, \ldots, n), l_{T_{i j}}^{y} l_{I_{i j}}^{y} l_{F_{i j}}^{y} \in L$. Then, we can get the neutrosophic linguistic decision evaluation matrix $R^{y}$, which is shown in Table 1.

Table 1. The neutrosophic linguistic decision matrix $R^{y}$ of the expert $E_{y}$.

\begin{tabular}{cccc}
\hline & $C_{1}$ & $\ldots$ & $C_{n}$ \\
\hline$A_{1}$ & $\left\langle l_{T_{11}}^{y}, l_{I_{11}}^{y}, l_{F_{11}}^{y}\right\rangle$ & $\ldots$ & $\left\langle l_{T_{1 n}}^{y}, l_{I_{1 n}}^{y}, l_{F_{1 n}}^{y}\right\rangle$ \\
$A_{2}$ & $\left\langle l_{T_{21}}^{y}, l_{I_{21}}^{y}, l_{F_{21}}^{y}\right\rangle$ & $\ldots$ & $\left\langle l_{T_{2 n},}^{y}, l_{I_{2 n},}^{y}, l_{F_{2 n}}^{y}\right\rangle$ \\
$\ldots$ & $\ldots$ & $\ldots$ & $\ldots$ \\
$A_{m}$ & $\left\langle l_{T_{m 1}}^{y}, l_{I_{m 1}}^{y}, l_{F_{m 1}}^{y}\right\rangle$ & $\ldots$ & $\left\langle l_{T_{m n}}^{y}, l_{I_{m n}}^{y}, l_{F_{m n}}^{y}\right\rangle$ \\
\hline
\end{tabular}

Then, based on the LNNNWBM or LNNNWGBM operator, we propose two decision-making methods, which are described as the following decision steps:

Step 1: According to the weight vector $w=\left(w_{1}, w_{2}, \ldots, w_{t}\right)^{T}$ of experts and the LNNNWBM operator, we can obtain the integrated matrix $R=\left(a_{i j}\right)_{m \times n^{\prime}}$, where the collective LNN $a_{i j}$ can be obtained by the following formula:

$$
\begin{aligned}
& a_{i, j}=\operatorname{LNNNWBM}\left(a_{i, j}^{1}, a_{i, j}^{2}, \ldots, a_{i, j}^{t}\right)=\left(\sum_{\substack{i, j=1 \\
j \neq i}}^{t} \frac{w_{i} w_{0} w_{i}}{1} a_{i} a_{i} a_{j}\right)^{\frac{1}{p+q}}
\end{aligned}
$$

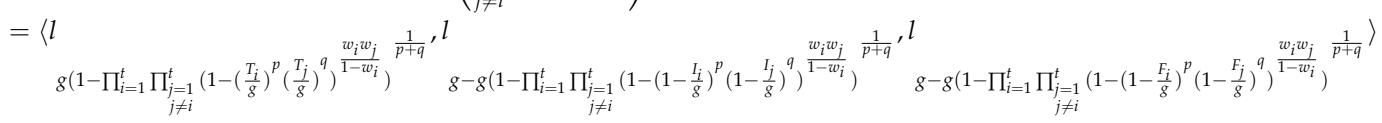

Step 2: According to the weight vector $\lambda=\left(\lambda_{1}, \lambda_{2}, \ldots, \lambda_{n}\right)^{T}$ of attributes and the LNNNWBM operator or the LNNNWGBM operator, we can obtain the total collective $\operatorname{LNN} a_{i}$ for $A_{i}(i=1,2, \ldots, m)$.

$$
\begin{aligned}
& a_{i}=\operatorname{LNNNWBM}\left(a_{i 1}, a_{i 2}, \ldots, a_{i n}\right)=\left(\sum_{\substack{j=1 \\
j \neq i}}^{n} \frac{w_{i} w_{j}}{1-w_{i}} a_{i}^{p} a_{j}\right)^{\frac{1}{p+q}}
\end{aligned}
$$

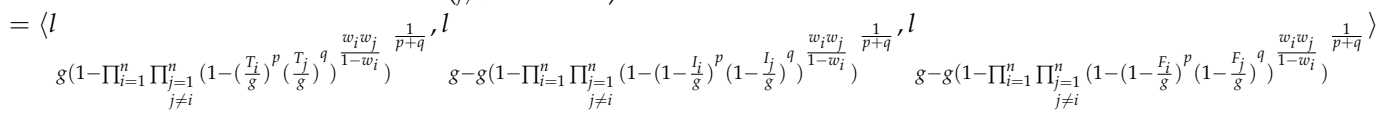


or:

$$
\begin{aligned}
& a_{i}=\operatorname{LNNNWGBM}\left(a_{i 1}, a_{i 2}, \ldots, a_{i n}\right)=\frac{1}{p+q} \otimes_{i=1}^{n} \otimes_{j=1, j \neq i}^{n}\left(p a_{i} \oplus q a_{j}\right)^{\frac{w_{i} w w_{j}}{1-w_{i}}}
\end{aligned}
$$

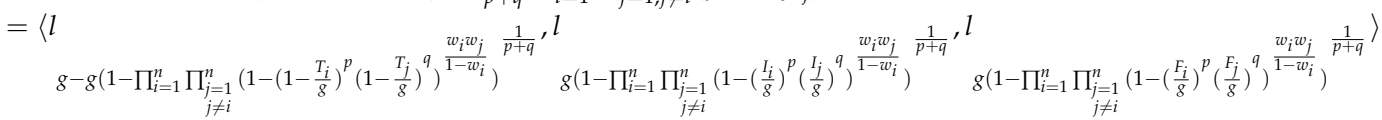

Step 3: According to the Equation (5) (Equation (6) if necessary), we calculate the expected value $E\left(a_{i}\right)$ and the accuracy $H\left(a_{i}\right)$ of the $\operatorname{LNN} A_{i}(i=1,2, \ldots, m)$.

Step 4: According to the value $E\left(a_{i}\right)\left(H\left(a_{i}\right)\right.$ if necessary), then we can rank the alternatives and choose the best one.

\section{Illustrative Examples}

The decision-making problem used in the literature [21] is considered here. There are four companies as a set of alternatives $A=\left\{A_{1}, A_{2}, A_{3}, A_{4}\right\}$, which are a car company $\left(A_{1}\right)$, a food company $\left(A_{2}\right)$, a computer company $\left(A_{3}\right)$, and an arm company $\left(A_{4}\right)$. An investment company needs to invest the best company, so they invite a set of three experts $E=\left\{E_{1}, E_{2}, E_{3}\right\}$ to evaluate these four companies. The evaluation of the alternatives must satisfy a set of three attributes $C=\left\{C_{1}, C_{2}, C_{2}\right\}$, which are the risk $\left(C_{1}\right)$, the growth $\left(C_{2}\right)$, and the environmental impact $\left(C_{3}\right)$. The importance of three experts is given as a weight vector $w=(0.37,0.33,0.3)^{T}$ and the importance of three attributes is given as a weight vector $\lambda=(0.35,0.25,0.4)^{T}$. Then, the evaluation criteria are based on the linguistic term set $L=\left\{l_{0}=\right.$ extremely bad, $l_{1}=$ very bad, $l_{2}=$ bad, $l_{3}=$ slightly bad, $l_{4}=$ medium, $l_{5}=$ slightly good, $l_{6}=$ good, $l_{7}=$ very good, $l_{8}=$ extremely good $\}$. Thus, we can establish the LNN decision matrix $R^{i}(i=1,2,3)$, which is listed in Tables $2-4$.

Table 2. The LNN decision matrix $R^{1}$ of the expert $E_{1}$.

\begin{tabular}{cccc}
\hline & $C_{\mathbf{1}}$ & $\boldsymbol{C}_{\mathbf{2}}$ & $\boldsymbol{C}_{\mathbf{3}}$ \\
\hline$A_{1}$ & $\left\langle l_{6}^{1}, l_{1}^{1}, l_{2}^{1}\right\rangle$ & $\left\langle l_{7}^{1}, l_{2}^{1}, l_{1}^{1}\right\rangle$ & $\left\langle l_{6}^{1}, l_{2}^{1}, l_{2}^{1}\right\rangle$ \\
$A_{2}$ & $\left\langle l_{7}^{1}, l_{1}^{1}, l_{1}^{1}\right\rangle$ & $\left\langle l_{7}^{1}, l_{3}^{1}, l_{2}^{1}\right\rangle$ & $\left\langle l_{7}^{1}, l_{2}^{1}, l_{1}^{1}\right\rangle$ \\
$A_{3}$ & $\left\langle l_{6}^{1}, l_{2}^{1}, l_{2}^{1}\right\rangle$ & $\left\langle l_{7}^{1}, l_{1}^{1}, l_{1}^{1}\right\rangle$ & $\left\langle l_{6}^{1}, l_{2}^{1}, l_{2}^{1}\right\rangle$ \\
$A_{4}$ & $\left\langle l_{7}^{1}, l_{1}^{1}, l_{2}^{1}\right\rangle$ & $\left\langle l_{7}^{1}, l_{2}^{1}, l_{3}^{1}\right\rangle$ & $\left\langle l_{7}^{1}, l_{2}^{1}, l_{1}^{1}\right\rangle$ \\
\hline
\end{tabular}

Table 3. The LNN decision matrix $R^{2}$ of the expert $E_{2}$.

\begin{tabular}{cccc}
\hline & $C_{1}$ & $C_{2}$ & $C_{3}$ \\
\hline$A_{1}$ & $\left\langle l_{6}^{2}, l_{1}^{2}, l_{2}^{2}\right\rangle$ & $\left\langle l_{6}^{2}, l_{1}^{2}, l_{1}^{2}\right\rangle$ & $\left\langle l_{4}^{2}, l_{2}^{2}, l_{3}^{2}\right\rangle$ \\
$A_{2}$ & $\left\langle l_{7}^{2}, l_{2}^{2}, l_{3}^{2}\right\rangle$ & $\left\langle l_{6}^{2}, l_{1}^{2}, l_{1}^{2}\right\rangle$ & $\left\langle l_{4}^{2}, l_{2}^{2}, l_{3}^{2}\right\rangle$ \\
$A_{3}$ & $\left\langle l_{5}^{2}, l_{1}^{2}, l_{2}^{2}\right\rangle$ & $\left\langle l_{5}^{2}, l_{1}^{2}, l_{2}^{2}\right\rangle$ & $\left\langle l_{5}^{2}, l_{4}^{2}, l_{2}^{2}\right\rangle$ \\
$A_{4}$ & $\left\langle l_{6}^{2}, l_{1}^{2}, l_{1}^{2}\right\rangle$ & $\left\langle l_{5}^{2}, l_{1}^{2}, l_{1}^{2}\right\rangle$ & $\left\langle l_{5}^{2}, l_{2}^{2}, l_{3}^{2}\right\rangle$ \\
\hline
\end{tabular}

Table 4. The LNN decision matrix $R^{3}$ of the expert $E_{3}$.

\begin{tabular}{cccc}
\hline & $C_{1}$ & $C_{2}$ & $C_{3}$ \\
\hline$A_{1}$ & $\left\langle l_{7}^{3}, l_{3}^{3}, l_{4}^{3}\right\rangle$ & $\left\langle l_{7}^{3}, l_{3}^{3}, l_{3}^{3}\right\rangle$ & $\left\langle l_{5}^{3}, l_{2}^{3}, l_{5}^{3}\right\rangle$ \\
$A_{2}$ & $\left\langle l_{6}^{3}, l_{3}^{3}, l_{4}^{3}\right\rangle$ & $\left\langle l_{5}^{3}, l_{1}^{3}, l_{2}^{3}\right\rangle$ & $\left\langle l_{6}^{3}, l_{2}^{3}, l_{3}^{3}\right\rangle$ \\
$A_{3}$ & $\left\langle l_{7}^{3}, l_{2}^{3}, l_{4}^{3}\right\rangle$ & $\left\langle l_{6}^{3}, l_{1}^{3}, l_{2}^{3}\right\rangle$ & $\left\langle l_{7}^{3}, l_{2}^{3}, l_{4}^{3}\right\rangle$ \\
$A_{4}$ & $\left\langle l_{7}^{3}, l_{2}^{3}, l_{3}^{3}\right\rangle$ & $\left\langle l_{5}^{3}, l_{2}^{3}, l_{1}^{3}\right\rangle$ & $\left\langle l_{6}^{3}, l_{1}^{3}, l_{1}^{3}\right\rangle$ \\
\hline
\end{tabular}




\subsection{The Decision-Making Process Based on the LNNNWBM Operator or LNNNWGBM Operator}

Step 1: According to the weight vector $w=(0.37,0.33,0.3)^{T}$ of experts and the LNNNWBM operator (set $p=1$ and $q=1$ ), we can obtain the integrated matrix $R=\left(a_{i j}\right)_{m \times n^{\prime}}$, which is listed in Table 5.

Table 5. The integrated matrix $R$.

\begin{tabular}{cccc}
\hline & $C_{1}$ & $C_{2}$ & $C_{3}$ \\
\hline$A_{1}$ & $\left\langle l_{6.3176}, l_{1.5682}, l_{2.6129}\right\rangle$ & $\left\langle l_{6.6819}, l_{1.9641}, l_{1.5682}\right\rangle$ & $\left\langle l_{5.0059}, l_{2.000}, l_{3.2898}\right\rangle$ \\
$A_{2}$ & $\left\langle l_{6.7045}, l_{1.9476}, l_{2.6308}\right\rangle$ & $\left\langle l_{6.0524}, l_{1.6728}, l_{1.6636}\right\rangle$ & $\left\langle l_{5.7033}, l_{2.000}, l_{2.3074}\right\rangle$ \\
$A_{3}$ & $\left\langle l_{5.9943}, l_{1.6636}, l_{2.6129}\right\rangle$ & $\left\langle l_{6.0264}, l_{1.000}, l_{1.6430}\right\rangle$ & $\left\langle l_{5.9943}, l_{2.6613}, l_{2.6129}\right\rangle$ \\
$A_{4}$ & $\left\langle l_{6.6819}, l_{1.2955}, l_{1.9641}\right\rangle$ & $\left\langle l_{5.6926}, l_{1.6636}, l_{1.6728}\right\rangle$ & $\left\langle l_{6.0264}, l_{1.6824}, l_{1.6170}\right\rangle$ \\
\hline
\end{tabular}

Step 2: According to the weight vector $\lambda=(0.35,0.25,0.4)^{T}$ of attributes and the LNNNWBM operator (set $p=1$ and $q=1$ ), we can obtain the collective overall LNNs of $a_{i}$ for $A_{i}(i=1,2,3,4$ ) as follows:

$$
\begin{gathered}
a_{1}=\left\langle l_{5.9328}, l_{1.8388}, l_{2.5784}\right\rangle, a_{2}=\left\langle l_{6.1489}, l_{1.8908}, l_{2.2399}\right\rangle, \\
a_{3}=\left\langle l_{6.0032}, l_{1.8430}, l_{2.3427}\right\rangle, \text { and } a_{4}=\left\langle l_{6.1675}, l_{1.5412}, l_{1.7536}\right\rangle .
\end{gathered}
$$

Step 3: Calculating the expected values of $E\left(a_{i}\right)$ for $a_{i}(i=1,2,3,4)$ :

$$
E\left(a_{1}\right)=0.7298, E\left(a_{2}\right)=0.7508, E\left(a_{3}\right)=0.7424 \text {, and } E\left(a_{4}\right)=0.7864 \text {. }
$$

According to the results, we can rank $E\left(a_{4}\right)>E\left(a_{2}\right)>E\left(a_{3}\right)>E\left(a_{1}\right)$, so the company $A_{4}$ is the best choice among all the companies.

On the other hand, we also use the LNNNWGBM operator (set $p=1$ and $q=1$ ) to deal with this decision-making problem:

Step 1': Just as step 1;

Step 2': According to the weight vector $\lambda=(0.35,0.25,0.4)^{T}$ of attributes and the LNNNWGBM operator (set $p=1$ and $q=1$ ), we can obtain the collective overall LNNs of $a_{i}$ for $A_{i}(i=1,2,3,4)$ as follows:

$$
\begin{gathered}
a_{1}=\left\langle l_{5.9970}, l_{1.8333}, l_{2.5434}\right\rangle, a_{2}=\left\langle l_{6.1790}, l_{1.8897}, l_{2.2324}\right\rangle, \\
a_{3}=\left\langle l_{6.0032}, l_{1.7928}, l_{2.3332}\right\rangle, \text { and } a_{4}=\left\langle l_{6.1824}, l_{1.5362}, l_{1.7500}\right\rangle .
\end{gathered}
$$

Step 3': Calculating the expected values of $E\left(a_{i}\right)$ for $a_{i}(i=1,2,3,4)$ :

$$
E\left(a_{1}\right)=0.7342, E\left(a_{2}\right)=0.7524, E\left(a_{3}\right)=0.7449 \text {, and } E\left(a_{4}\right)=0.7873 \text {. }
$$

According to the results, the ranking is $E\left(a_{4}\right)>E\left(a_{2}\right)>E\left(a_{3}\right)>E\left(a_{1}\right)$, so the company $A_{4}$ is the best choice among all the companies.

\subsection{Analysis the Influence of the Parameters $p$ and $q$ on Decision Results}

In order to analyze the effects of different parameters $p$ and $q$ on the decision results, in Steps 1 and 2, we take the different values of $p$ and $q$, and all the results are shown in the Tables 6 and 7 . 
Table 6. The ranking based on the LNNNWBM operator with the different values of $p$ and $q$.

\begin{tabular}{cll}
\hline$p, q$ & \multicolumn{1}{c}{ LNNNWBM Operator } & Ranking \\
\hline$p=1, q=0$ & $E\left(a_{1}\right)=0.7528, E\left(a_{2}\right)=0.7777, E\left(a_{3}\right)=0.7613, E\left(a_{4}\right)=0.8060$ & $A_{4} \succ A_{2} \succ A_{3} \succ A_{1}$ \\
$p=1, q=0.5$ & $E\left(a_{1}\right)=0.7311, E\left(a_{2}\right)=0.7534, E\left(a_{3}\right)=0.7435, E\left(a_{4}\right)=0.7886$ & $A_{4} \succ A_{2} \succ A_{3} \succ A_{1}$ \\
$p=1, q=2$ & $E\left(a_{1}\right)=0.7329, E\left(a_{2}\right)=0.7545, E\left(a_{3}\right)=0.7453, E\left(a_{4}\right)=0.7897$ & $A_{4} \succ A_{2} \succ A_{3} \succ A_{1}$ \\
$p=0, q=0$ & $E\left(a_{1}\right)=0.7573, E\left(a_{2}\right)=0.7766, E\left(a_{3}\right)=0.7656, E\left(a_{4}\right)=0.8046$ & $A_{4} \succ A_{2} \succ A_{3} \succ A_{1}$ \\
$p=0.5, q=1$ & $E\left(a_{1}\right)=0.7326, E\left(a_{2}\right)=0.7530, E\left(a_{3}\right)=0.7449, E\left(a_{4}\right)=0.7879$ & $A_{4} \succ A_{2} \succ A_{3} \succ A_{1}$ \\
$p=2, q=1$ & $E\left(a_{1}\right)=0.7349, E\left(a_{2}\right)=0.7562, E\left(a_{3}\right)=0.7463, E\left(a_{4}\right)=0.7902$ & $A_{4} \succ A_{2} \succ A_{3} \succ A_{1}$ \\
$p=2, q=2$ & $E\left(a_{1}\right)=0.7343, E\left(a_{2}\right)=0.7537, E\left(a_{3}\right)=0.7458, E\left(a_{4}\right)=0.7884$ & $A_{4} \succ A_{2} \succ A_{3} \succ A_{1}$ \\
\hline
\end{tabular}

Table 7. The ranking based on the LNNNWGBM operator with the different values of $p$ and $q$.

\begin{tabular}{cll}
\hline $\mathbf{p}, \mathbf{q}$ & LNNNWGBM Operator & Ranking \\
\hline$p=1, q=0$ & $E\left(a_{1}\right)=0.7397, E\left(a_{2}\right)=0.7747, E\left(a_{3}\right)=0.7531, E\left(a_{4}\right)=0.8035$ & $A_{4} \succ A_{2} \succ A_{3} \succ A_{1}$ \\
$p=1, q=0.5$ & $E\left(a_{1}\right)=0.7342, E\left(a_{2}\right)=0.7545, E\left(a_{3}\right)=0.7453, E\left(a_{4}\right)=0.7891$ & $A_{4} \succ A_{2} \succ A_{3} \succ A_{1}$ \\
$p=1, q=2$ & $E\left(a_{1}\right)=0.7343, E\left(a_{2}\right)=0.7548, E\left(a_{3}\right)=0.7457, E\left(a_{4}\right)=0.7889$ & $A_{4} \succ A_{2} \succ A_{3} \succ A_{1}$ \\
$p=0, q=1$ & $E\left(a_{1}\right)=0.7437, E\left(a_{2}\right)=0.7730, E\left(a_{3}\right)=0.7570, E\left(a_{4}\right)=0.8019$ & $A_{4} \succ A_{2} \succ A_{3} \succ A_{1}$ \\
$p=0.5, q=1$ & $E\left(a_{1}\right)=0.7356, E\left(a_{2}\right)=0.7541, E\left(a_{3}\right)=0.7467, E\left(a_{4}\right)=0.7885$ & $A_{4} \succ A_{2} \succ A_{3} \succ A_{1}$ \\
$p=2, q=1$ & $E\left(a_{1}\right)=0.7330, E\left(a_{2}\right)=0.7553, E\left(a_{3}\right)=0.7445, E\left(a_{4}\right)=0.7895$ & $A_{4} \succ A_{2} \succ A_{3} \succ A_{1}$ \\
$p=2, q=2$ & $E\left(a_{1}\right)=0.7334, E\left(a_{2}\right)=0.7530, E\left(a_{3}\right)=0.7441, E\left(a_{4}\right)=0.7877$ & $A_{4} \succ A_{2} \succ A_{3} \succ A_{1}$ \\
\hline
\end{tabular}

From above two tables, we can see that when the parameters $p$ and $q$ take different values, the sorting results are the same. Therefore, the influence of the two parameters is very little in this decision-making problem.

In the literature [21], the ranking is $A_{4} \succ A_{2} \succ A_{3} \succ A_{1}$, just according with the ranking result of this paper. Compared with the literature [21], the correlation between attributes is considered by the LNNNWBM operator and the LNNNWGBM operator for MAGDM, which make the information aggregation more objective and reliable. Hence, the proposed MAGDM methods with different $p$ and $q$ values are more flexible than the method in [21]. Compared to the literature [14], on the one hand, the literature [14] cannot express and deal with the decision-making problems with pure linguistic information like LNNs. However, in this paper, the proposed decision-making methods based on the $L N N N W B M$ operator and the LNNNWGBM operator provide a new way for decision-makers under LNN environment.

\section{Conclusions}

In MADGM, how to tackle the problem of the interdependence between attributes is a challenging issue. Thus, MADGM methods based on the LNNNWGBM and LNNNWGBM operators for LNNs are proposed in this paper. First, a LNN normalized weight Bonferroni mean (LNNNWBM) operator and a LNN normalized weight geometric Bonferroni mean (LNNNWGBM) operator are proposed based on the BM operator, and the related properties of these operators are discussed. Second, based on the LNNNWBM operator and the LNNNWGBM operator, this paper puts forward two methods of MADGM in a LNN setting. Finally, an illustrative example was presented to show that these two methods were used for solving the MADGM problem with LNN information. In addition, the proposed decision-making methods may affect the decision results based on various parameters of $p$ and $q$ in some decision-making problems.

Acknowledgments: This work was supported by the National Natural Science Foundation of China under grant Nos. 61603258 and 61703280.

Author Contributions: Changxing Fan originally proposed the LNNNWBM and LNNNWGBM operators and investigated their properties; Jun Ye, Keli Hu and En Fan provided the calculation and comparative analysis; and we wrote the paper together.

Conflicts of Interest: The author declares no conflict of interest. 


\section{References}

1. Zadeh, L.A. The concept of a linguistic variable and its application to approximate reasoning-I. Inf. Sci. 1975, 8, 199-249. [CrossRef]

2. Zadeh, L.A. A concept of a linguistic variable and its application to approximate reasoning-II. Inf. Sci. 1975, 8, 301-357. [CrossRef]

3. Herrera, F.; Herrera-Viedma, E. Linguistic decision analysis: Steps for solving decision problems under linguistic information. Fuzzy Sets Syst. 2000, 115, 67-82. [CrossRef]

4. Herrera, F.; Herrera-Viedma, E. A model of consensus in group decision making under linguistic assessments. Fuzzy Sets Syst. 1996, 78, 73-87. [CrossRef]

5. Liu, P.D. Some generalized dependent aggregation operators with intuitionistic linguistic numbers and their application to group decision making. J. Comput. Syst. Sci. 2013, 79, 131-143. [CrossRef]

6. Chen, Z.C.; Liu, P.D.; Pei, Z. An approach to multiple attribute group decision making based on linguistic intuitionistic fuzzy numbers. Int. J. Comput. Intell. Syst. 2015, 8, 747-760. [CrossRef]

7. Liu, P.D.; Teng, F. An extended TODIM method for multiple attribute group decision-making based on 2-dimension uncertain linguistic variable. Complexity 2016, 21, 20-30. [CrossRef]

8. Liu, P.D.; Li, H.; Yu, X.C. Generalized hybrid aggregation operators based on the 2-dimension uncertain linguistic information for multiple attribute group decision making. Group Decis. Negot. 2016, 25, 103-126. [CrossRef]

9. Liu, P.D.; Wang, P. Some improved linguistic intuitionistic fuzzy aggregation operators and their applications to multiple-attribute decision making. Int. J. Inf. Technol. Decis. Mak. 2017, 16, 817-850. [CrossRef]

10. Smarandache, F. Neutrosophy: Neutrosophic Probability, Set, and Logic; American Research Press: Rehoboth, DE, USA, 1998.

11. Smarandache, F. A unifying field in logics. In Neutrosophy: Neutrosophic Probability, Set and Logic; American Research Press: Rehoboth, DE, USA, 1999.

12. Wang, H.; Smarandache, F.; Zhang, Y.Q.; Sunderraman, R. Single valued neutrosophic sets. Multispace Multistruct. 2010, 4, 410-413.

13. Ye, J. An extended TOPSIS method for multiple attribute group decision making based on single valued neutrosophic linguistic numbers. J. Intell. Fuzzy Syst. 2015, 28, 247-255.

14. Liu, P.D.; Shi, L.L. Some neutrosophic uncertain linguistic number Heronian mean operators and their application to multi-attribute group decision making. Neural Comput. Appl. 2017, 28, 1079-1093. [CrossRef]

15. Bonferroni, C. Sulle medie multiple di potenze. Bollettino dell'Unione Matematica Italiana 1950, 5, $267-270$. (In Italian)

16. Zhou, W.; He, J.M. Intuitionistic fuzzy normalized weighted Bonferroni mean and its application in multicriteria decision making. J. Appl. Math. 2012, 2012, 1-22. [CrossRef]

17. Zhu, B.; Xu, Z.S.; Xia, M.M. Hesitant fuzzy geometric Bonferroni means. Inf. Sci. 2012, 205, 72-85. [CrossRef]

18. Sun, M.; Liu, J. Normalized geometric Bonferroni operators of hesitant fuzzy sets and their application in multiple attribute decision making. J. Inf. Comput. Sci. 2013, 10, 2815-2822. [CrossRef]

19. Liu, P.D.; Chen, S.M.; Liu, J.L. Some intuitionistic fuzzy interaction partitioned Bonferroni mean operators and their application to multi-attribute group decision making. Inf. Sci. 2017, 411, 98-121. [CrossRef]

20. Liu, P.D.; Li, H.G. Interval-valued intuitionistic fuzzy power Bonferroni aggregation operators and their application to group decision making. Cogn. Comput. 2017, 9, 494-512. [CrossRef]

21. Fang, Z.B.; Ye, J. Multiple Attribute Group Decision-Making Method Based on Linguistic Neutrosophic Numbers. Symmetry 2017, 9, 111. [CrossRef]

(C) 2017 by the authors. Licensee MDPI, Basel, Switzerland. This article is an open access article distributed under the terms and conditions of the Creative Commons Attribution (CC BY) license (http:/ / creativecommons.org/licenses/by/4.0/). 